\title{
Radiation induces epithelial-mesenchymal transition in colorectal cancer cells
}

\author{
AYA KAWAMOTO $^{1}$, TAKESHI YOKOE ${ }^{1}$, KOJI TANAKA ${ }^{1}$, SUSUMU SAIGUSA $^{1}$, YUJI TOIYAMA ${ }^{1}$, \\ HIROMI YASUDA $^{1}$, YASUHIRO INOUE ${ }^{1}$, CHIKAO MIKI $^{1}$ and MASATO KUSUNOKI ${ }^{1,2}$
}

Departments of ${ }^{1}$ Gastrointestinal and Pediatric Surgery, and ${ }^{2}$ Innovative Surgery, Division of Reparative Medicine, Institute of Life Sciences, Mie University Graduate School of Medicine, Tsu, Mie 514-8507, Japan

Received July 5, 2011; Accepted August 30, 2011

DOI: 10.3892/or.2011.1485

\begin{abstract}
Radiotherapy remains a major approach to adjuvant therapy for patients with advanced rectal cancer. Nevertheless, the effects of radiation on malignant processes have yet to be clarified. The aim of this study was to assess the biological effects of radiation on colorectal cancer (CRC) cells with special reference to epithelial-mesenchymal transition (EMT), a key developmental program often activated during cancer invasion and metastasis. We investigated the effect of radiation on two colorectal cancer cell lines, CaR1 and DLD1, assessing cell morphology, motility, migration and invasive ability. Expression of molecules associated with EMT was determined using RT-PCR, Western blotting, and immunofluorescence staining in control and irradiated cells. We also used real-time RT-PCR to examine the expression of molecules associated with EMT before and after chemoradiotherapy. Thus, we studied 26 rectal cancer patients who received preoperative chemoradiotherapy followed by radical surgery. In addition, we examined the relationship between disease recurrence and the expression of a number of proteins. Irradiation caused CRC cells to undergo phenotypic changes characteristic of EMT: spindle-cell shape, loss of polarity, intercellular separation and pseudopodia formation. Irradiation enhanced cell migration and invasiveness. In irradiated CRC cells, molecular changes consistent with EMT were observed. In clinical samples, we observed molecular changes consistent with EMT, and those changes were significantly enhanced in patients with recurring disease. These results indicate that irradiation induces an alteration to a malignant phenotype consistent with EMT in colorectal cancer cells.
\end{abstract}

Correspondence to: Dr Aya Kawamoto, Department of Gastrointestinal and Pediatric Surgery, Division of Reparative Medicine, Institute of Life Sciences, Mie University Graduate School of Medicine, Tsu, Mie 514-8507, Japan

E-mail: ayayaka@clin.medic.mie-u.ac.jp

Key words: epithelial-mesenchymal transition, radiation, rectal cancer

\section{Introduction}

Rectal cancer is one of the most common and deadly cancers in developed countries, accounting for approximately $8 \%$ of deaths from all cancers worldwide (1). Since the incidence of local recurrence following curative resection ranges between 4 and $32 \%$ with a median of over $15 \%$ (2), efforts to improve the prognosis of rectal cancer have focused on controlling local recurrence. Introduction of radiotherapy, chemoradiotherapy and improvements in operative procedures such as total mesorectal excision (TME) has enhanced both local pelvic control and survival. In particular, adjuvant radiotherapy followed by surgery has been widely used in an attempt to improve treatment of rectal cancer, and it has become a standard therapeutic strategy in the Western world. The aim of preoperative radiotherapy is to sterilize the resection margins and to destroy microscopic collections of cancer cells situated outside the mesorectum, in presacral lymph nodes and at lateral pelvic walls, a potential focus of local recurrence and, a source of distant metastasis. Previous studies using a high dose radiation with variable fraction schedules have demonstrated a significant reduction in local recurrence compared with surgery alone (3-5). However, a significant number of patients suffer from local recurrence and distant metastasis even after multi-modality therapy. Thus, adjuvant chemoradiotherapy is still unable to achieve high rates of survival.

Previous researchers have observed that treatment of the primary tumor with either surgery or radiotherapy can have unpredictable effects on metastasis. Although radiotherapy is a major therapeutic modality for cancer treatment, previous findings indicate that irradiation promotes tumor migration, distant metastasis, and the invasive potential of cancer cells (6-8). Irradiation activates multiple signaling pathways (9) and affects the activity or abundance of proteases, growth factors, cytokines and adhesion proteins that are involved in tissue remodeling (10). The first major experiment examining the effect of local irradiation on the frequency of metastasis was performed by Kaplan and Murphy in 1949 (11). Their study was based on the clinical observation that several patients with epidermoid carcinoma of the lip who had received prior radiotherapy presented to them with widely metastatic disease. In 2001, Camphausen et al reported that radiation therapy administered to a primary Lewis lung carcinoma accelerated 
metastatic growth in mice (8). In that study, the authors observed that irradiating a primary tumor induced a net imbalance of pro-angiogenic over anti-angiogenic factors within the metastatic vascular bed, and rapid expansion followed. Several studies have reported on the radiation-enhanced invasiveness of colorectal cancer, and the increased expression of MMP has been considered as a mechanism for the increase in invasive capacity (12-14). Despite many investigations, the effects of irradiation on malignant biological behaviors have yet to be fully clarified.

On the other hand, the process of cell migration and invasion during metastasis is frequently associated with the loss of epithelial markers and the acquisition of mesenchymal markers as well as increased migration and motility, cellular changes which are known as the epithelial-mesenchymal transition (EMT). Recent reports have suggested that EMT is induced by irradiation in several solid cancers, but not in colorectal cancer (CRC).

In the present study, we examined the effect of irradiation on the morphology, motility and invasiveness of colorectal cancer cells, and also examined whether this alteration was associated with EMT.

\section{Materials and methods}

Cell culture. Two human colorectal cancer cell lines, CaR1 and DLD1, were used. The cell lines were obtained from the Medical Institute of Bioregulation, Kyushu University (Oita, Japan). CaR-1 cells were maintained in Dulbecco's modified Eagle's medium (DMEM), and DLD1 cells were maintained in 45\% RPMI-1640 medium, supplemented with $10 \% \mathrm{v} / \mathrm{v}$ fetal bovine serum (FBS; Gibco-BRL, Tokyo, Japan), penicillin (100,000 units/l), streptomycin (100 mg/l), and gentamicin $(40 \mathrm{mg} / \mathrm{l})$ and incubated at $37^{\circ} \mathrm{C}$ in a $5 \% \mathrm{CO}_{2}$ environment.

Chemoradiation procedure in vitro. Colorectal cancer cells were irradiated at room temperature. Cells were treated with 5 Gy of X-ray irradiation (100 kV and $3.5 \mathrm{~mA}$ ) using an X-ray generator (MBR-1505R; Hitachi Medical Co., Tokyo, Japan).

RNA extraction. Total RNA was extracted from each of the cell lines and clinical materials, and purified using an RNeasy Mini Kit (Qiagen, Tokyo, Japan) according to the manufacturer's protocol.

Quantitative real-time PCR. Total RNA was extracted from 24-h post-infection cell lysates using RNeasy kits with DNase I treatment as directed by the manufacturer. The total RNA in each sample was quantified by absorbance readings at 260 nm using a Beckman-Coulter DU 530 spectrophotometer. Following RNA purification, PCR analysis was performed using $50 \mathrm{ng}$ total RNA in reactions containing Taqman ${ }^{\circledR}$ One-step RT-PCR Master Mix Kit and gene-specific primers for epithelial cadherin (E-cadherin) $(C D H I)$, vimentin (VIM), fibronectin 1 (FN1), snail (SNAI1), slug (SNAI2), and twist (TWIST1). Amplification was performed in an ABI Prism 7900 HT Sequence Detector using standard settings of 40 cycles with an annealing temperature of $60^{\circ} \mathrm{C}$. All samples were amplified in five parallel reactions, and experiments were performed at least twice with similar results.
Relative gene expression levels of each target gene. Relative gene expression levels were determined by the standard curve method. The standard curves and linear equations were generated using 5-fold serially diluted solutions of cDNA from qPCR Human Reference Total RNA (Clontech, Mountain View, CA) for each gene. All standard curves were linear in the analyzed range with an acceptable correlation coefficient $\left(R^{2}\right)$. The amount of target gene expression was calculated from the standard curve followed by quantitative normalization of cDNA in each sample using $A C T B$ gene expression as an internal control. Target gene mRNA levels are provided as ratios to $A C T B$ mRNA levels. RT-PCR assays were carried out in duplicate for each sample, and the mean value was used for calculation of the mRNA expression levels.

Wound assay. Cells were grown in 10-cm culture dishes. When the cells reached confluency, the medium was replaced with fresh RPMI-1640 containing 10\% FBS. Confluent cells were wounded with a uniform scratch using sterile pipette tips, rinsed to remove debris, then incubated in culture medium containing 10\% FBS for $18 \mathrm{~h}$. Quantitative analysis of the wound healing index expressed as a percentage was calculated using 20 randomly chosen distances across the wound at 0 and $18 \mathrm{~h}$, divided by the distance measured at $0 \mathrm{~h}$.

Invasion assay. Cell invasiveness was evaluated using 24-well Matrigel invasion chambers (Becton-Dickinson Labware). Cells were suspended in the upper chamber at a final concentration of $10 \times 10^{4} / \mathrm{ml}$ in $200 \mu \mathrm{l}$ of RPMI-1640 supplemented with $1 \%$ FBS. The lower chamber contained $700 \mu 1$ of RPMI1640 supplemented with $1 \%$ FBS. After 22-h of incubation, the tumor cells remaining on the upper surface of each filter were removed by wiping with a cotton swab, and the invading cells on the lower surface were stained with Diff-Quik stain. The number of cells on the lower surface of each filter was counted under a microscope at a magnification of $x 200$. The invasion assay was performed in triplicate in three individual experiments.

Western blot analysis. Plates were washed in ice-cold PBS, after which cold lysis buffer (Tris-buffered saline, $\mathrm{pH} 7.5$, containing $1 \%$ Triton X-100) was added directly to the plates. Cells were scraped from plates, collected, and homogenized by using a Mixer Mill MM 300 homogenizer (Qiagen Inc., Chatsworth, CA, USA). Supernatants were collected and frozen at $-20^{\circ} \mathrm{C}$ until use. The protein concentration was measured by the BCA protein assay (Pierce, Rockford, IL, USA). Lysate proteins $(20 \mu \mathrm{g})$ were mixed with an equal volume of $2 \mathrm{X}$ Laemmli's loading buffer containing $2-\mathrm{ME}$ and heated at $100^{\circ} \mathrm{C}$ for $5 \mathrm{~min}$. Samples were electrophoretically separated on $12.5 \%$ gradient polyacrylamide gels containing $0.1 \%$ SDS, followed by semi-dry transfer to an Immun-Blot PVDF membrane (Bio-Rad Laboratories, Hercules, CA, USA). The membranes were blocked using 5\% skim milk in Tris-buffered saline, $\mathrm{pH} 7.5$, supplemented with $0.1 \%$ Tween-20 (TBS-T). We used anti-E-cadherin, anti-vimentin, and anti- $\beta$-actin antibodies. The primary antibodies were washed in $0.05 \%$ Tween-20/PBS and then incubated with horseradish peroxidase-conjugated secondary antibody. Chemiluminescent signals were visualized 
A

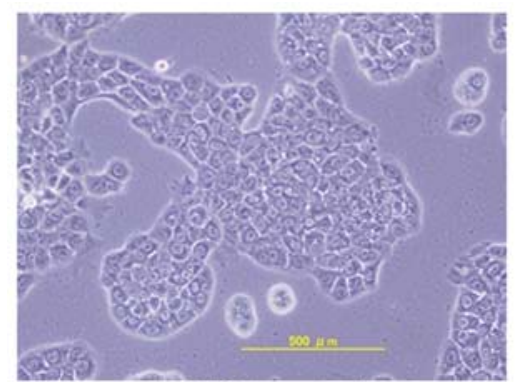

CaR1 control

B

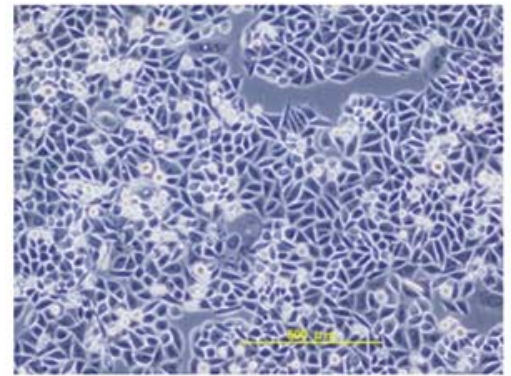

DLD1 control

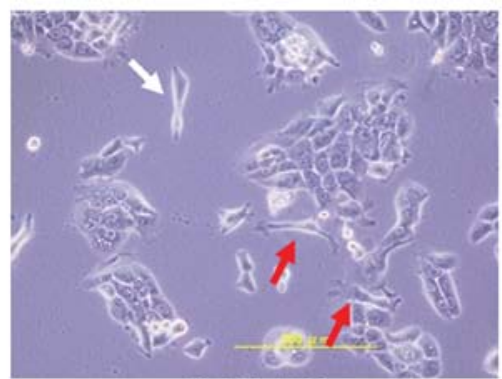

CaR1 5 Gy

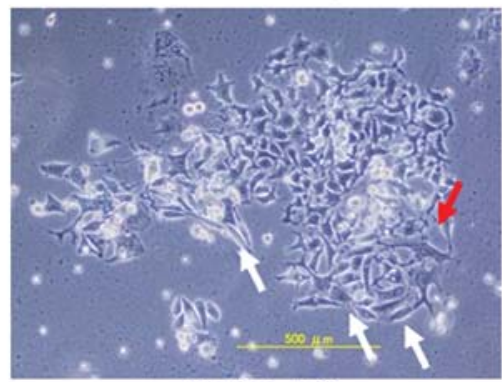

DLD1 5 Gy

Figure 1. Irradiation induces morphological changes consistent with EMT in CaR1 (A) and DLD1 (B) cells. Spindle-shaped cells showed a loss of polarity (white arrows), and pseudopodia (red arrows) were noted in the cells after irradiation in both cell lines.

in a CS Analyzer and AE-6962 light capture system (ATTO Corp., Tokyo, Japan).

Clinical materials. A total of 26 patients with rectal cancer were enrolled in the study. The patients ranged in age from 48 to 77 years (mean age, 64.1 years). All patients received preoperative chemoradiotherapy. They were treated with external irradiation (10 megavoltage photons from a linear accelerator) using a 4-field box technique and received $20 \mathrm{~Gy}$ in four fractions in one week. They also underwent concurrent chemotherapy (combination of intravenous infusion of 5-fluorouracil and oral administration of uracil and tegafur) over one week. The rectal tumors were biopsied by endoscopy for histopathological diagnosis before irradiation. Two or three biopsy specimens were obtained and examined after informed consent was obtained from the patients. All tumors were diagnosed as adenocarcinoma, and the patients underwent chemoradiotherapy, followed by surgery. Fresh frozen samples were also obtained from operative specimens as postchemoradiation samples.

Statistical analysis. The results were assessed by paired t-test and $\chi^{2}$ test; $p<0.05$ was considered statistically significant. All statistical analyses were performed using JMP software.

\section{Results}

Irradiation induces morphological changes consistent with EMT in CaRl and DLDl cells. The phenotypic changes observed in the irradiated rectal cancer cell line, CaR1, included loss of cell polarity, appearance of spindle-shaped cells, and increased formation of pseudopodia (Fig. 1A), which are consistent with the mesenchymal phenotype. Irradiation induced similar morphologic changes in the colon cancer cell line, DLD1 (Fig. 1B).
Table I. Changes in target gene expression in CaR1 and DLD1 cells after irradiation.

\begin{tabular}{lcccccc}
\hline & E-cadherin & Vimentin & FN1 & Snail & Slug & Twist \\
\hline CaR1 & 0.476 & 1.593 & 2.943 & 1.462 & 4.564 & 0.878 \\
DLD1 & 0.617 & 1.513 & 1.182 & 1.134 & 3.535 & 0.737 \\
\hline
\end{tabular}

The numerical values show the ratio of gene expression in nonirradiated and irradiated cell lines.

Increased migration and invasive capacity in irradiated CaRI and DLD1 cells. EMT is characterized by increased motility and gain of invasiveness. To determine whether irradiated cells had become more active in migration and invasion than non-irradiated cells, we evaluated serum-induced migration of cells using Transwell chamber assays. As shown in Fig. 2A, $\sim 25 \%$ more irradiated CaR1 cells migrated through the pores than did non-irradiated CaR1 cells. The invasive ability of irradiated and non-irradiated cells was examined by seruminduced invasion through a Transwell extracellular matrix layer. As shown in Fig. 2B, irradiated CaR1 cells exhibited a 6-fold increase in invasion compared to non-irradiated CaR1 cells after a 24-h incubation. The enhanced migration was also confirmed by wound healing assays conducted for $40 \mathrm{~h}$ (Fig. 3). Similar results were observed in irradiated and nonirradiated DLD1 cells.

Irradiated CaRI and DLD1 cells exhibit molecular changes consistent with EMT. To determine whether irradiation induces specific molecular changes consistent with EMT, we performed real-time PCR (Table I). Expression of the epithelial adhesion molecule E-cadherin $(\mathrm{CDHI})$ was decreased in irradiated 
A

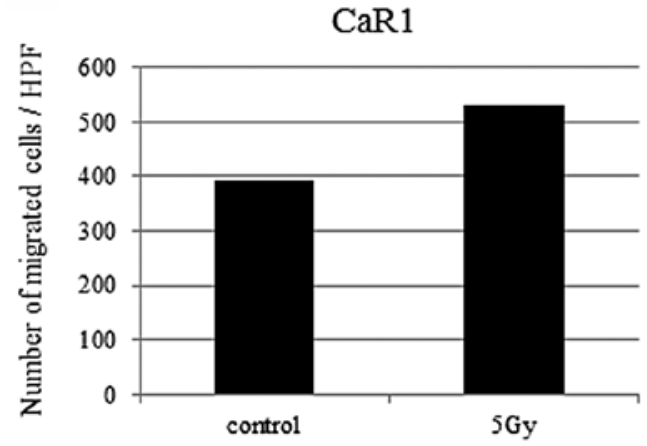

B

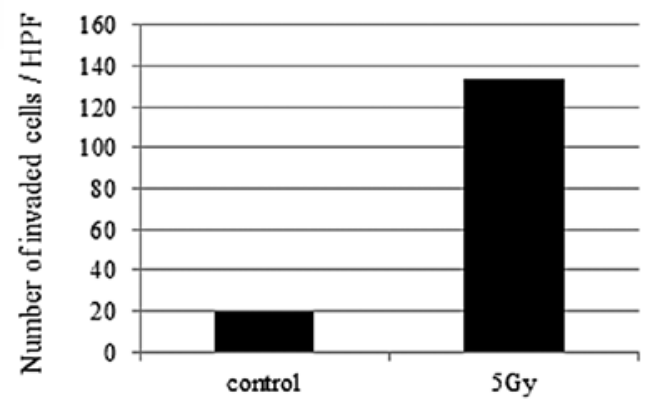

DLD1

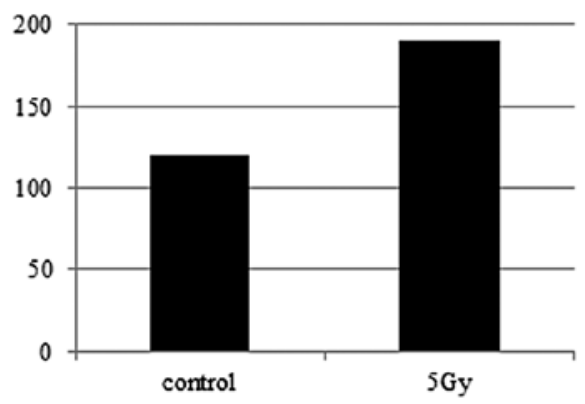

DLD1

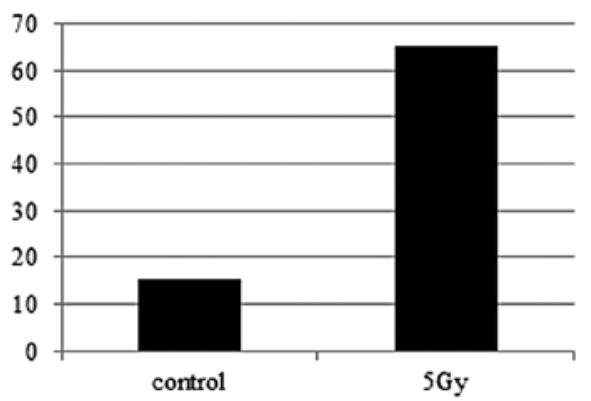

Figure 2. Irradiated cells demonstrate increased migratory and invasive capacities. (A) After 24 h, irradiated cells exhibited an $\sim 1.2-$ to 1.5 -fold increase in the number of cells migrating through the collagen insert. (B) After $24 \mathrm{~h}$, irradiated cells exhibited an $\sim 5$ - to 10-fold increase in the number of cells invading through the Matrigel-coated collagen insert.

cells compared with the non-irradiated cells. Increases in the expression of the mesenchymal markers, vimentin (VIM) and fibronectin $1(F N 1)$ were also observed. Furthermore, increased expression of the EMT-related transcription factor slug (SNAI2) was observed in irradiated CRC cells compared with parental cells. No significant changes were observed in levels of the EMT-related transcription factors snail (SNAII) and twist (TWIST1).

Western blotting was carried out to assess E-cadherin and vimentin protein levels to determine whether irradiation induces changes consistent with EMT (Fig. 4A). Expression of E-cadherin was decreased in irradiated cells compared with the non-irradiated cells, and increases in the expression of vimentin were also observed. To investigate the possibility that irradiation leads to changes in the cellular localization of E-cadherin, immunofluorescence staining was conducted using CaR1 control and irradiated cells (Fig. 4B). Irradiated cells exhibited a change in E-cadherin from an organized, membrane-bound structure to a disorganized state throughout the cytoplasm.

Cancer tissues show molecular changes consistent with EMT after chemoradiotherapy (CRT)

Patient characteristics. To confirm the biological effect of irradiation on CRC patients, 25 patients were included in this study. The median age was 61.5 years (range, $41-78$ years); the male to female ratio was 7.2:1. The median follow-up period was 39 months (range, 11-75 months). The pre-CRT clinical T stages were as follows: T2, 30.7\% $(\mathrm{n}=8) ; \mathrm{T} 3,50 \%$ $(n=13) ;$ T4, 19.2\% $(n=5)$. The post-CRT pathological stages were $\mathrm{T} 1,19.2 \%(\mathrm{n}=5) ; \mathrm{T} 2,19.2 \%(\mathrm{n}=5) ; \mathrm{T} 3,57.6 \%(\mathrm{n}=15) ; \mathrm{T} 4$, $3.8 \%(n=1)$. Twelve patients $(46.1 \%)$ had pathological lymph node metastases. Lymphatic invasion was present in 18 of 26 patients $(69.2 \%)$, and vascular invasion was present in 14 patients (53.8\%). Six patients developed recurrence; 4 patients developed distant metastasis, and 2 patients developed local recurrence.

To determine whether CRT induces specific molecular changes consistent with EMT, we measured the expression of $C D H 1$ as an epithelial marker, VIM, FN1, as mesenchymal markers, SNAI1, SNAI2 and TWIST1 as EMT-related transcription factors in matched pre- and post-CRT specimens (Fig. 5). CDH1 was significantly decreased in post-CRT specimens $(\mathrm{p}=0.0065)$. On the other hand, VIM, FN1 were significantly increased after CRT ( $<<0.0001$ and $\mathrm{p}=0.0002$, respectively). SNAI1, SNAI2 and TWIST1 were also significantly increased after CRT. Furthermore, as shown in Table II, patients who exhibited decreased levels of $\mathrm{CDH} 1$ developed recurrence at a significantly greater frequency $(n=6)$. In this study, no significance was observed in increased levels of mesenchymal markers (VIM, FNI) and SNAI1, SNAI2, TWIST1 in patients who developed recurrence. Both $C D H 1$ down-regulation and VIM up-regulation were significantly frequently observed in patients who developed recurrence.

\section{Discussion}

EMT was initially observed in embryonic development during which cells lose epithelial characteristics and gain mesenchymal properties to increase motility and invasiveness $(15,16)$. Recent research suggests that EMT plays a central role in tumor progression and metastasis (15-18), and is induced by growth factors implicated in these processes, including hepatocyte growth factor, transforming growth factor $\beta$ (TGF- $\beta$ ), 


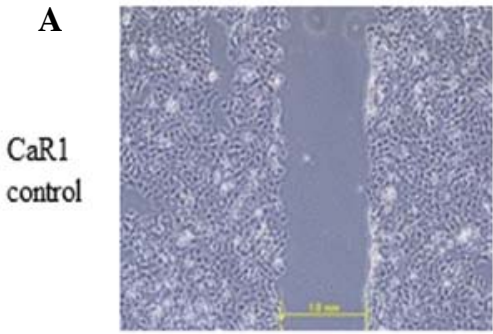

$\mathrm{Oh}$

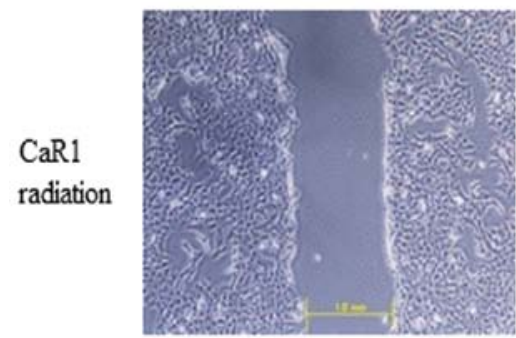

Oh

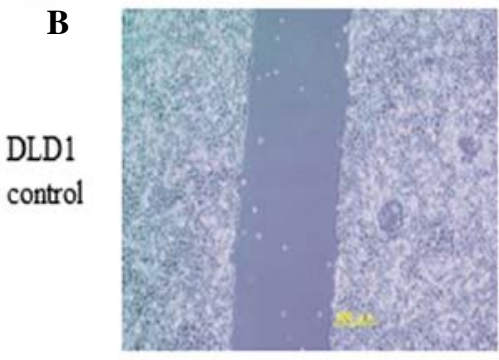

Oh

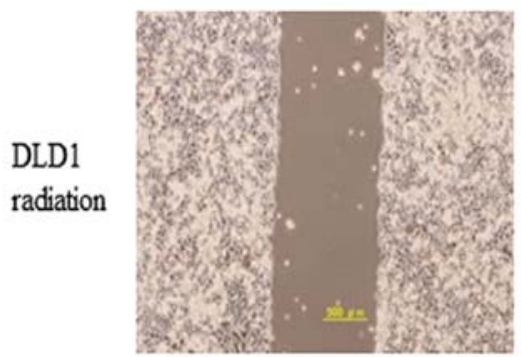

Oh

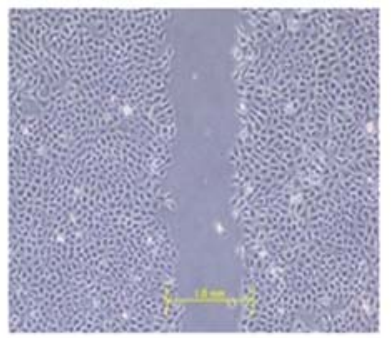

$20 \mathrm{~h}$

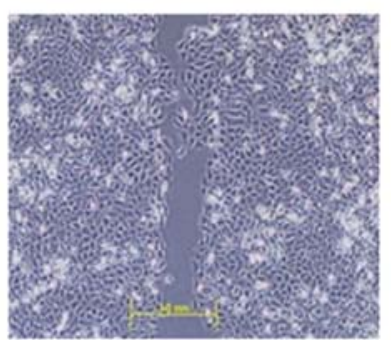

$20 \mathrm{~h}$

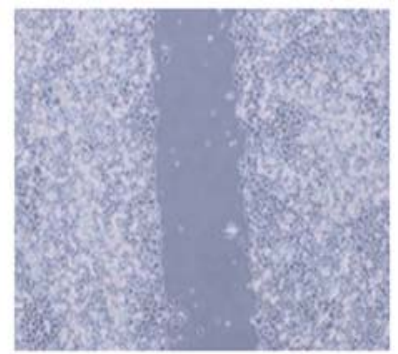

$20 \mathrm{~h}$

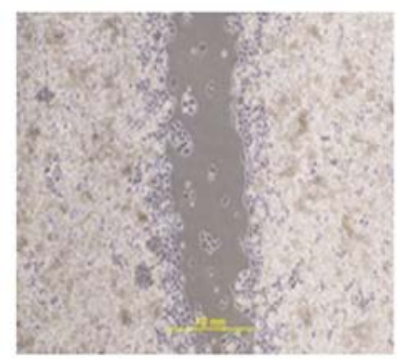

$20 \mathrm{~h}$

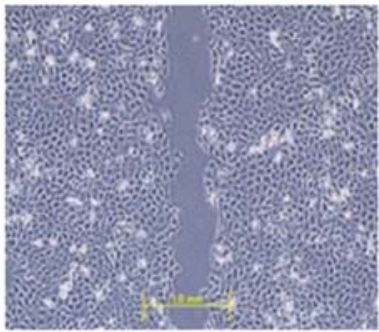

$40 \mathrm{~h}$

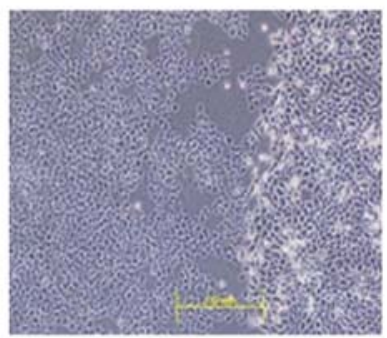

$40 \mathrm{~h}$

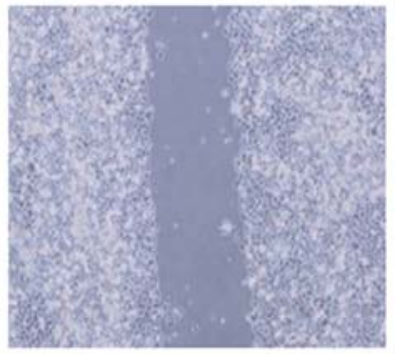

$40 \mathrm{~h}$

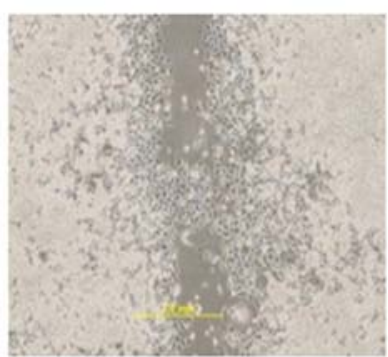

$40 \mathrm{~h}$

Figure 3. Wound closure assays of DLD1 and CaR1 cells treated or not treated with radiation (5 Gy). Representative images captured with a x10 objective at the time of wounding $(0 \mathrm{~h})$ and 20 or $40 \mathrm{~h}$ after wounding. All experiments were repeated at least three times with similar results.

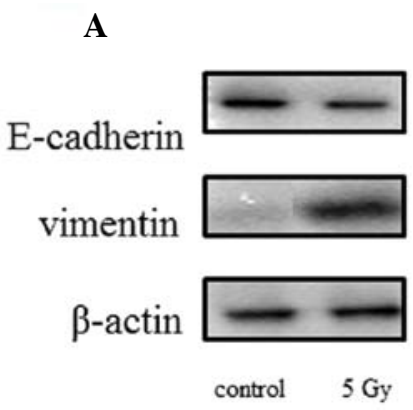

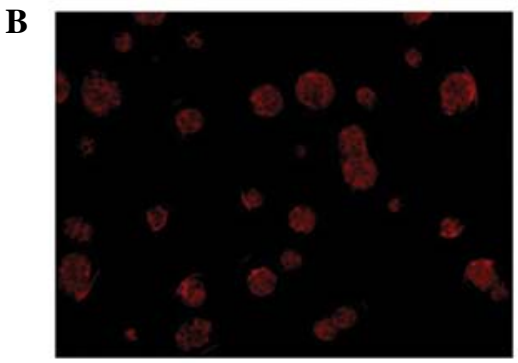

control

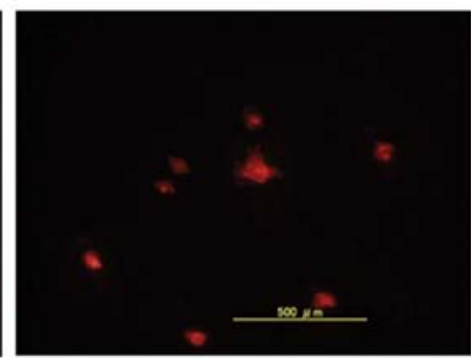

$5 \mathrm{~Gy}$

Figure 4. Irradiation induces molecular changes consistent with EMT. (A) Cell lysates and nuclear extracts from CaR1 control and irradiated cells were subjected to Western blotting. Expression of the epithelial cellular adhesion molecule E-cadherin was decreased. In contrast, the expression of the mesenchymal marker vimentin was increased in irradiated cells compared with control cells. (B) Immunofluorescence analysis of CAR1 cells for E-cadherin. Irradiated cells showed changes in the localization of E-cadherin from their usual cell membrane-associated site. E-cadherin was observed in a disorganized cytoplasmic location. 

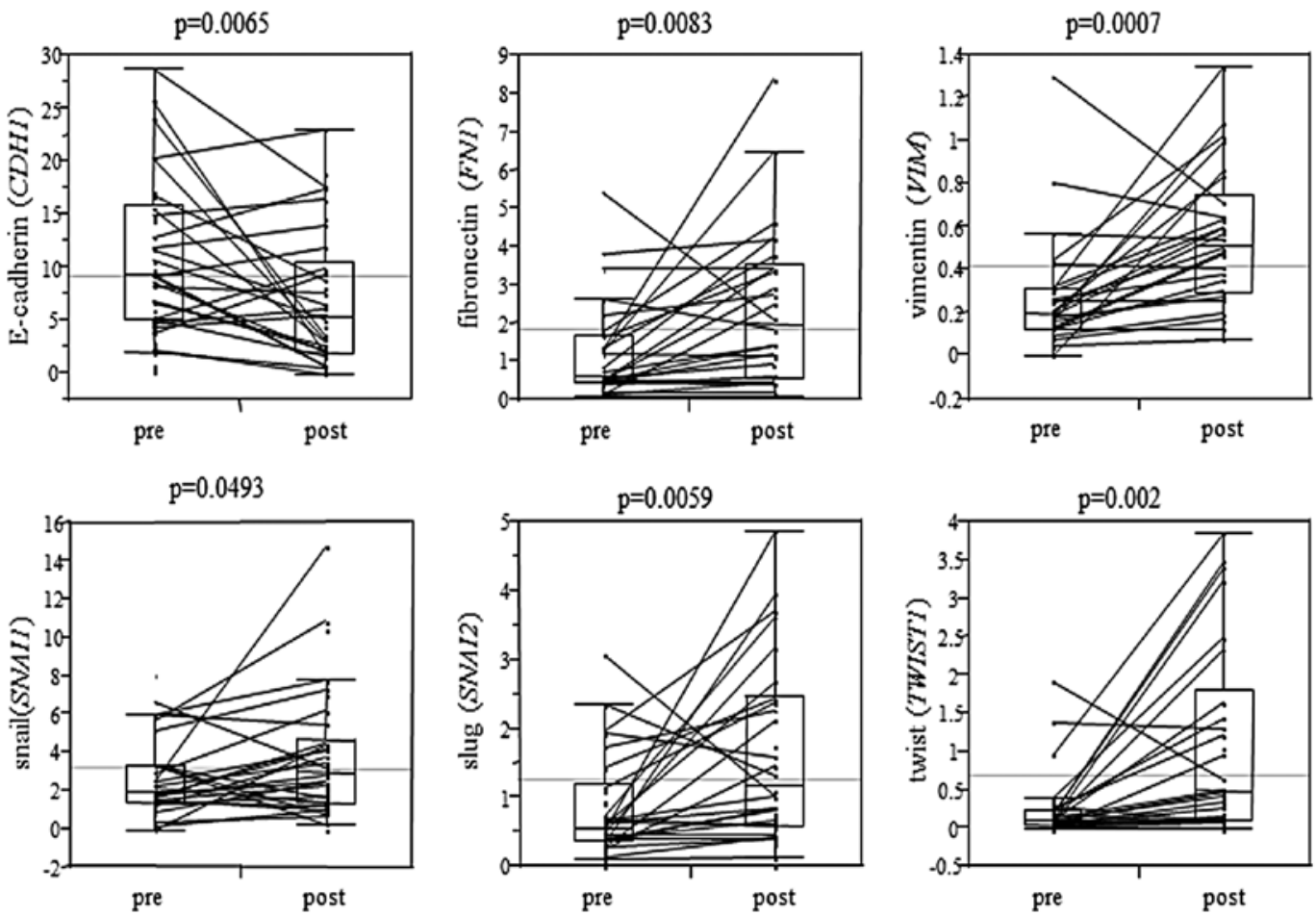

Figure 5. Rectal cancer tissues demonstrate molecular changes consistent with EMT after CRT. Twenty-six tissue samples were obtained from rectal cancer patients who received chemoradiotherapy followed by radical surgery. We measured the expression of E-cadherin as an epithelial marker, vimentin, fibronectin as mesenchymal markers, snail, slug, and twist as EMT-related transcription factors in matched pre- and post-CRT specimens. E-cadherin was significantly decreased in post-CRT specimens $(\mathrm{p}=0.0065)$. In contrast, vimentin, fibronectin were significantly increased during $\mathrm{CRT}$ ( $\mathrm{p}<0.0001$ and $\mathrm{p}=0.0002$, respectively).

Table II. Changes in target gene expression consistent with EMT were enhanced in patients who developed recurrence.

\begin{tabular}{lcrl}
\hline & Recurence $(+)$ & Recurrence $(-)$ & p-value \\
\hline E-cad $\uparrow$ & 6 & 11 & 0.04214 \\
E-cad $\downarrow$ & 0 & 9 & \\
Vim $\uparrow$ & 5 & 15 & 0.67090 \\
Vim $\downarrow$ & 1 & 5 & \\
FN1 $\uparrow$ & 5 & 14 & 0.43041 \\
FN1 $\downarrow$ & 1 & 7 & \\
Snail $\uparrow$ & 5 & 13 & 0.09717 \\
Snail $\downarrow$ & 0 & 8 & \\
Slug $\uparrow$ & 5 & 15 & 0.55731 \\
Slug $\downarrow$ & 1 & 6 & \\
Twist $\uparrow$ & 0 & 4 & 0.31266 \\
Twist $\downarrow$ & 4 & 15 & \\
E-cad $\downarrow /$ Vim $\uparrow$ & 5 & 7 & 0.04691 \\
Others & 1 & 12 & \\
\hline
\end{tabular}

and epidermal growth factor (19). In primary tumors, the induction of EMT can lead to structural changes involving cell adhesion molecules, and in particular E-cadherin (20-22). E-cadherin is a transmembrane glycoprotein that is localized in the adherens junction typically found in epithelial cells, and plays an important role in maintaining the structural integrity of epithelial sheets. Several EMT-inducing regulators that repress E-cadherin have been described. The major proteins implicated in the transcriptional repression of E-cadherin include the zinc finger proteins snail and slug, $\delta E F$, smad interacting protein 1, ZEB2, and a basic helixloop-helix (bHLH) protein called twist. Several signaling pathways are also reported to be associated with EMT, which lead to transcriptional and post-transcriptional induction of EMT transcription factors (23). In regard to the relationship between irradiation and EMT, Andarawewa et al reported that irradiation induced TGF- $\beta$-mediated EMT (24).

Several studies have examined the possible role of EMT in CRC progression $(25,26)$, and the association between chemoresistance and EMT in CRC (27-29). On the other hand, Kumar et al $(12,13)$ and Speake et al $(14)$ and described that irradiation enhanced the migration and invasiveness of CRC cells in association with enhanced expression and activity of MMPs, but no studies have referred to radiation-induced EMT in CRC cells.

In the present study, we observed that irradiation enhanced the invasive potential of CRC cells, and also demonstrated that this irradiation-enhanced invasiveness was associated with morphological and molecular alterations consistent with a change to a mesenchymal-like phenotype. Furthermore, we confirmed a similar phenomenon in clinical rectal cancer specimens.

In our results, irradiation enhanced cell adhesion, and increased both motility and invasive capacity. These changes are all associated with the cellular transition from epithelial to fibroblastoid phenotype, characterized by enhanced cell 
motility. In other words, irradiation enhanced the invasiveness of CRC cells, partially through morphological and molecular changes resembling EMT. In clinical samples, we also observed molecular changes consistent with EMT and those changes were significantly enhanced in recurrent cases. This observation suggests that it is necessary to examine the mechanism of the relationship between irradiation and EMT in rectal cancer patients to improve survival further.

In conclusion, irradiation induces cells to adopt a malignant phenotype, a process consistent with EMT in CRC cells. The suppression of the molecules associated with EMT represents a potentially effective strategy for the treatment of CRC patients who have undergone chemoradiotherapy, although further investigation is needed.

\section{References}

1. Parkin DM, Bray F, Ferlay J and Pisani P: Global cancer statistics, 2002. CA Cancer J Clin 55: 74-108, 2005.

2. Abulafi AM and Williams NS: Local recurrence of colorectal cancer: the problem, mechanisms, management and adjuvant therapy. Br J Surg 81: 7-19, 1994.

3. Marsh PJ, James RD and Schofield PF: Adjuvant preoperative radiotherapy for locally advanced rectal carcinoma. Results of a prospective, randomized trial. Dis Colon Rectum 37: 1205-1214, 1994.

4. Cedermark B, Johansson H, Rutqvist LE and Wilking N: The Stockholm I trial of preoperative short term radiotherapy in operable rectal carcinoma. A prospective randomized trial. Stockholm Colorectal Cancer Study Group. Cancer 75: 2269-2275, 1995.

5. Improved survival with preoperative radiotherapy in resectable rectal cancer. Swedish Rectal Cancer Trial. N Engl J Med 336 980-987, 1997.

6. Wild-Bode C, Weller M, Rimner A, Dichgans J and Wick W: Sublethal irradiation promotes migration and invasiveness of glioma cells: implications for radiotherapy of human glioblastoma. Cancer Res 61: 2744-2750, 2001.

7. Qian LW, Mizumoto K, Urashima T, et al: Radiation-induced increase in invasive potential of human pancreatic cancer cells and its blockade by a matrix metalloproteinase inhibitor, CGS27023. Clin Cancer Res 8: 1223-1227, 2002.

8. Camphausen K, Moses MA, Beecken WD, Khan MK, Folkman J and O'Reilly MS: Radiation therapy to a primary tumor accelerates metastatic growth in mice. Cancer Res 61: 2207-2211, 2001.

9. Dent P, Yacoub A, Contessa J, et al: Stress and radiation-induced activation of multiple intracellular signaling pathways. Radiat Res 159: 283-300, 2003.

10. Barcellos-Hoff MH, Park C and Wright EG: Radiation and the microenvironment - tumorigenesis and therapy. Nat Rev Cancer 5: 867-875, 2005.

11. Kaplan HD and Murphy ED: The effect of local roentgen irradiation on the biological behavior of a transplantable mouse carcinoma; increased frequency of pulmonary metastasis. J Nat Cancer Inst 9: 407-413, 1949.
12. Kumar A, Collins HM, Scholefield JH and Watson SA: Increased type-IV collagenase (MMP-2 and MMP-9) activity following preoperative radiotherapy in rectal cancer. $\mathrm{Br} \mathrm{J}$ Cancer 82: 960-965, 2000

13. Kumar A, Collins H, Van Tam J, Scholefield JH and Watson SA: Effect of preoperative radiotherapy on matrilysin gene expression in rectal cancer. Eur J Cancer 38: 505-510, 2002.

14. Speake WJ, Dean RA, Kumar A, Morris TM, Scholefield JH and Watson SA: Radiation induced MMP expression from rectal cancer is short lived but contributes to in vitro invasion. Eur J Surg Oncol 31: 869-874, 2005.

15. Hay ED: The mesenchymal cell, its role in the embryo, and the remarkable signaling mechanisms that create it. Dev Dyn 233: 706-720, 2005.

16. Thiery JP: Epithelial-mesenchymal transitions in tumour progression. Nat Rev Cancer 2: 442-454, 2002.

17. Thiery JP and Sleeman JP: Complex networks orchestrate epithelial-mesenchymal transitions. Nat Rev Mol Cell Biol 7: 131-142, 2006.

18. Lee JM, Dedhar S, Kalluri R and Thompson EW: The epithelialmesenchymal transition: new insights in signaling, development, and disease. J Cell Biol 172: 973-981, 2006.

19. Elliott BE, HungWL, Boag AH and Tuck AB: The role of hepatocyte growth factor (scatter factor) in epithelial-mesenchymal transition and breast cancer. Can J Physiol Pharmacol 80: 91-102, 2002.

20. Ikeguchi M, Makino $M$ and Kaibara N: Clinical significance of E-cadherin-catenin complex expression in metastatic foci of colorectal carcinoma. J Surg Oncol 77: 201-207, 2001.

21. Takeichi M: Cadherins in cancer: implications for invasion and metastasis. Curr Opin Cell Biol 5: 806-811, 1993.

22. Birchmeier $\mathrm{W}$ and Behrens $\mathrm{J}$ : Cadherin expression in carcinomas: role in the formation of cell junctions and the prevention of invasiveness. Biochim Biophys Acta 1198: 11-26, 1994.

23. Sabbah M, Emami S, Redeuilh G, et al: Molecular signature and therapeutic perspective of the epithelial-to-mesenchymal transitions in epithelial cancers. Drug Resist Updat 11: 123-151, 2008.

24. Andarawewa KL, Erickson AC, Chou WS, et al: Ionizing radiation predisposes non-malignant human mammary epithelial cells to undergo transforming growth factor beta-induced epithelial to mesenchymal transition. Cancer Res 67: 8662-8670, 2007.

25. Bates RC, Bellovin DI, Brown C, et al: Transcriptional activation of integrin beta 6 during the epithelial-mesenchymal transition defines a novel prognostic indicator of aggressive colon carcinoma. J Clin Invest 115: 339-347, 2005.

26. Brabletz T, Hlubek F, Spaderna S, Schmalhofer O, Hiendlmeyer E, Jung A and Kirchner T: Invasion and metastasis in colorectal cancer: epithelial-mesenchymal transition, mesenchymal-epithelial transition, stem cells and beta-catenin. Cells Tissues Organs 179: 56-65, 2005.

27. Nakamura T, Kato Y, Fuji H , Horiuchi T, Chiba Y and Tanaka K: E-cadherin-dependent intercellular adhesion enhances chemoresistance. Int J Mol Med 12: 693-700, 2003.

28. St Croix B and Kerbel RS: Cell adhesion and drug resistance in cancer. Curr Opin Oncol 9: 549-556, 1997.

29. Yang AD, Fan F, Camp ER, et al: Chronic oxaliplatin resistance induces epithelial-to-mesenchymal transition in colorectal cancer cell lines. Clin Cancer Res 12: 4147-4153, 2006. 